\title{
Support Vector machine and duration-aware conditional random field for identification of spatio-temporal activity patterns by combined indoor positioning and heart rate sensors
}

\author{
Jan Behmann ${ }^{1}$ • Kathrin Hendriksen ${ }^{2}$ - Ute Müller ${ }^{3}$. \\ Wolfgang Büscher ${ }^{2} \cdot$ Lutz Plümer $^{1}$
}

Received: 24 October 2014 / Revised: 3 April 2016 /

Accepted: 26 April 2016 / Published online: 18 May 2016

(C) The Author(s) 2016. This article is published with open access at Springerlink.com

\begin{abstract}
Tracking the spatio-temporal activity is highly relevant for domains like security, health, and quality management. Since animal welfare became a topic in politics and legislation locomotion patterns of livestock have received increasing interest. In contrast to the monitoring of pedestrians cattle activity tracking poses special challenges to both sensors and data analysis. Interesting states are not directly observable by a single sensor. In addition, sensors must be accepted by cattle and need to be robust enough to cope with a rough environment. In this article, we introduce the novel combination of heart rate and positioning sensors. Attached to neck and chest they are less interfering than accelerometers at the ankles. Exploiting the potential of such combined sensor system that records locomotion and nonspatial information from the heart rate sensor however is challenging. We introduce a novel two level method for the activity tracking focused on the duration and sequence of activity states. We combine Support Vector Machine (SVM) with Conditional Random Field (CRF) and extend Conditional Random fields by an explicit representation of duration. The SVM characterizes local activity states, whereas the CRF addresses sequences of local states to sequences incorporating spatial and non-spatial contextual knowledge. This combination provides a reliable and comprehensive identification of defined activity patterns, as well as their chronology and durations, suitable for the integration in an activity data base. This data
\end{abstract}

Jan Behmann

behmann@igg.uni-bonn.de

1 Department of Geoinformation, Institute of Geodesy and Geoinformation, University of Bonn, Bonn, Germany

2 Department of Livestock Technology, Institute of Agricultural Engineering, University of Bonn, Bonn, Germany

3 Department of Physiology \& Hygiene, Institute of Animal Science, University of Bonn, Bonn, Germany 
base is used to extract physiological parameters and promises insights into internal states such as fitness, well-being and stress. Interestingly we were able to demonstrate a significant correlation between resting pulse rate and the day of pregnancy.

Keywords Spatio-temporal pattern · Machine learning $\cdot$ Activity monitoring $\cdot$ Indoor positioning system · Animal monitoring

\section{Introduction}

The analysis of trajectories of so called Moving Point Objects (MPO) is relevant for many important applications [1-3]. One goal of the interpretation of locomotion patterns is the identification and temporal positioning of cattle activities. The focus of this paper is a method for the robust identification of activities based on two sensors, one spatial (position) and one non-spatial (heart rate). Whereas the limited accuracy of the indoor positioning sensor in the third dimension does not allow a reliable distinction between lying and standing, the additional information from the heart rate sensor does. Thus in the proposed approach, the non-spatial sensor supports the identification of activities with locomotion and, in addition, enables the identification of states without locomotion.

For measuring the spatial trajectories of moving objects multiple sensor principles are available. In many cases, such trajectories are recorded by mobile phones with self-locating functionality [4] or cameras [5], but in specific local scenarios indoor positioning systems are applied [6]. These spatial sensors generate time series of coordinates which were analyzed for activity information [7]. Interpreting such trajectories is defined as deriving semantic information from the geometric features, meaning generating high-level knowledge from low-level data [8].

In this study, we evaluate the data from the novel integration of positioning system and heart rate sensor to observe dairy cattle with minimal interfering. We apply a novel integration of Support Vector Machines with Conditional Random Fields including an explicit representation of the duration. The distinction between states such as lying and standing is difficult since the positioning system provides no z-coordinates. Our approach provides an added value for research in cattle welfare by an automated and reliable identification of relevant activity patterns. It goes beyond unconnected time stamps and was designed for a reliable estimation of sequence and duration of activity states by robust sensors. The two sensor types complement each other in recording information about the activity patterns and internal states.

In dairy research there is a general interest in analyzing the heart rate and, especially, the heart rate variability to get access to wellbeing and stress [9]. However, without a reliable distinction between resting and activity the analysis of the heart rate data is difficult.

The data set containing the trajectories and heart signals of dairy cows was used to derive a sequence of activity states. The map of the barn and the samples of two simultaneously recorded signals including the corresponding activity states are shown in Fig. 1. Different approaches for the interpretation of movement patterns have been published $[10,11]$. Some methods were developed for unconstrained movement, e.g. the routes of migratory birds [11] or the scale-dependency of cow trajectories [12]. In recent time, machine learning methods like SVM [13, 14] and CRF [15] are increasingly adopted. However, in many scenarios available spatial constraints, e.g. road graphs or the border of playing field, improve the position accuracy and simplify the interpretation. 


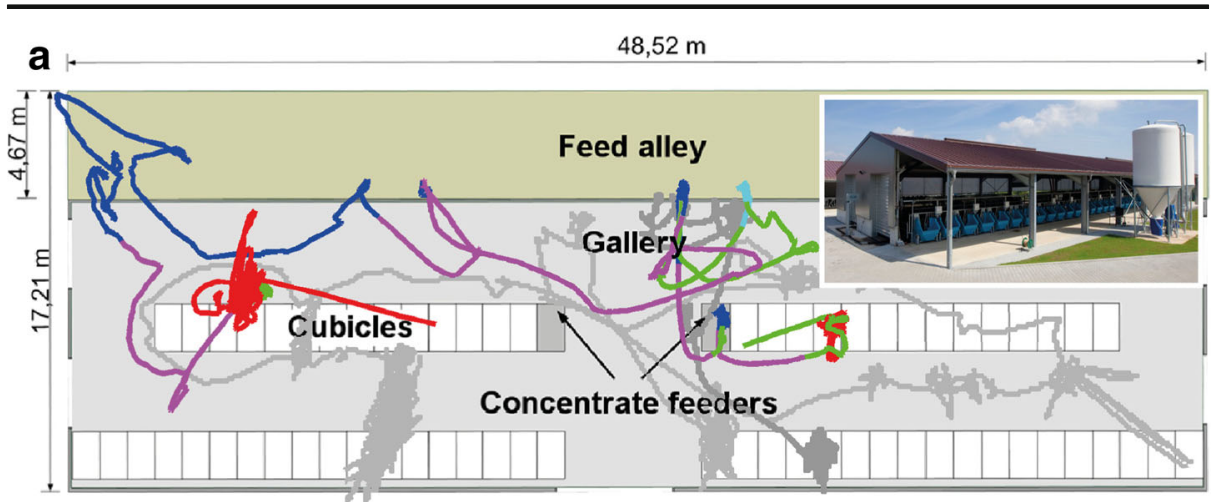

b

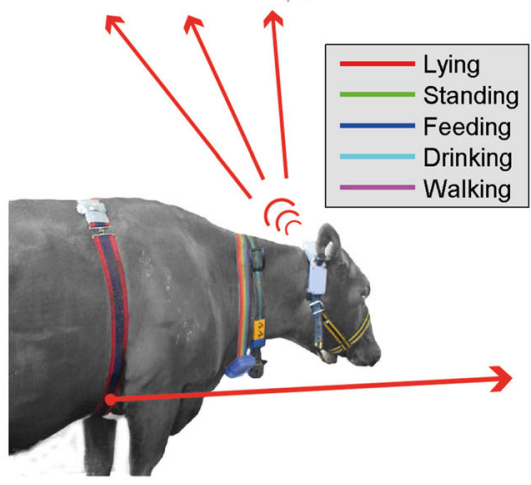

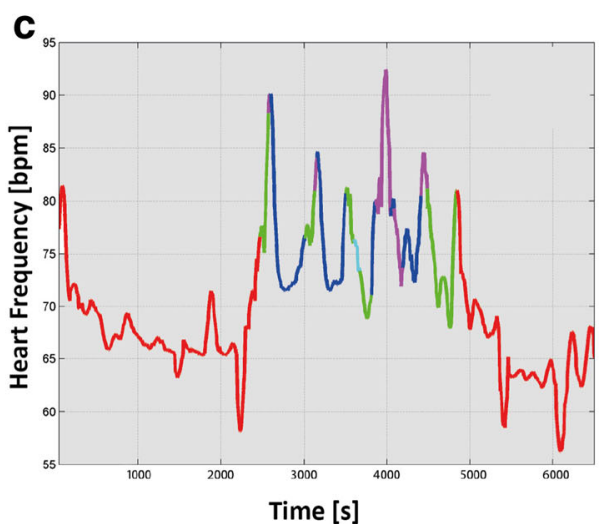

Time [s]

Fig. 1 The used sensors attached to a cow: The Local Positioning Measurement System attached to the neck belt and the heart rate sensor under the chest strap b. A filtered sample in both signal types with corresponding activity states: the Local Position Measurement (LPM) system in the research barn a and the heart frequency c. The map of the barn visualizes two additional trajectories to imply potential investigations of social interactions

Furthermore, known areas or points of interests, like train stations or feeding station at animal monitoring support the reliable interpretation [10].

However, the interpretation of movement patterns, whether in a local or a global context, is not sufficient for the detection of activities without or with only small movements below the resolution of the positioning system. Such activities require further information recorded by additional sensors. Therefore, the integration of heterogeneous data from multiple sensors including different measuring frequencies, scales and reference systems is required. Such an approach is still a challenge for data analysis methods.

In recent years in European countries, especially in Germany, there is a vivid debate on intensive livestock farming, and the pressure on farmers ensuring animal welfare is increasing. The big question is how to identify, objectify and quantify animal welfare, and how to provide optimal conditions in stable design and management for both, welfare and performance. To this end continuous monitoring and interpretation of the derived data is becoming increasingly important. Animal behavior represented by sequences of activities and locomotion patterns provide sensitive indicators for this kind of research.

Several studies have used accelerometers to monitor animal movement patterns $[16,17]$. Martiskainen et al. [18] used the data from a three-dimensional accelerometer and multiclass support vector machine classifiers to develop a method for measuring behavior patterns. 
Although this method achieves a high prediction quality, information regarding the cow position in $3 \mathrm{D}$ space and its relative position to other objects like feeding tables or cubicles are absent. In the open air, it is possible to track the movement patterns of the animals using the signals of the Global Positioning System (GPS [19]). Early approaches to continuous monitoring of cattle with multiple sensors were discussed in [20].

However, under a roof, the usefulness of such systems is limited since obstacles (walls, ceilings, etc.) weaken the signals [21]. For indoor application a tracking system based on electromagnetic waves, such as the ABATEC System [22] has been used (Fig. 1). It is a local position measurement (LPM) system developed for field sports [6] and can determine the positions of moving objects in real time. This system achieves an accuracy which is superior to GPS and specified by the manufacturer with a standard deviation of $2 \mathrm{~cm}$ [22]. However, for measurements in a barn a standard deviation of up to $20 \mathrm{~cm}$ was reported in [23]. Neisen et al. [24] used the LPM System to monitor cow behavior and movement patterns. In a cow barn, shadings and reflections resulting from metal surfaces and the relatively small distances between the antennas greatly impair sensor signals (Fig. 1). The signal-to-noise ratio declines, the outlier rate exceeds $10 \%$ and the height coordinate ceases [23]. These data characteristics complicate the reliable analysis of recorded trajectories.

A promising approach to get reliable activity information even from such unreliable position signals is the integration of additional sensors in the analysis. The heart rate and the heart rate variability are strongly related to animal welfare [9] and are strong indicators for the physical stress of the body. As summarized by Langbein et al. [25] the heart rate indicates changes of the sympathetic tone, without considering parasympathetic influences. On the other hand, parasympathetic influences and changes in vagal activity are registered by the heart rate variability (HRV). To compare processes (or animals) with each other, heart rate data are related to specific states [26]. States like lying, in which the heart rate of the individual is relatively stable and which are less affected by short-term influences, are best suited for the interpretation of the cardiac response of an animal.

The interpretation of heart signals is challenging as these signals represent simultaneously various processes, short- and long-term as well as relevant and irrelevant ones. A promising approach for defining heart rate properties is the use of Recurrence Quantitative Analysis (RQA) - this specifies the characteristics of nonlinear systems [27]. Mohr et al. [28] used the RQA to assess stress levels in calves and cows based on the heart rate variability Non-invasive and, thus non-stress-inducing techniques like heart rate measurements enable the assessment of stress levels without having an effect on the heart rate parameters itself [29].

We present a prediction method that uses signals from spatial (LPM) and non-spatial (heart rate) sensors for the prediction of activity state sequences (Fig. 2). In the domain of machine learning this kind of problem is solved by sequence labeling algorithms [30]. These algorithms have been successfully applied in DNA sequencing [31], natural speech recognition [32] and protein structure prediction [33]. These tasks require on the one hand the interpretation of signals and on the other hand, the modeling of the temporal neighborhood within the label sequence. A common characteristic of these methods is the use of graphical models [34] often in the form of a Hidden Markov Model (HMM [35]). This approach has been extended and improved to the discriminative modeling realized by the Conditional Random Field (CRF [36]). In contrast do HMMs, CRFs do not require strong assumption on statistical independence which are unlikely in most cases and thus provide more reliable predictions. In Machine Learning, esp. image processing and robotics, they have become very popular in recent years. 


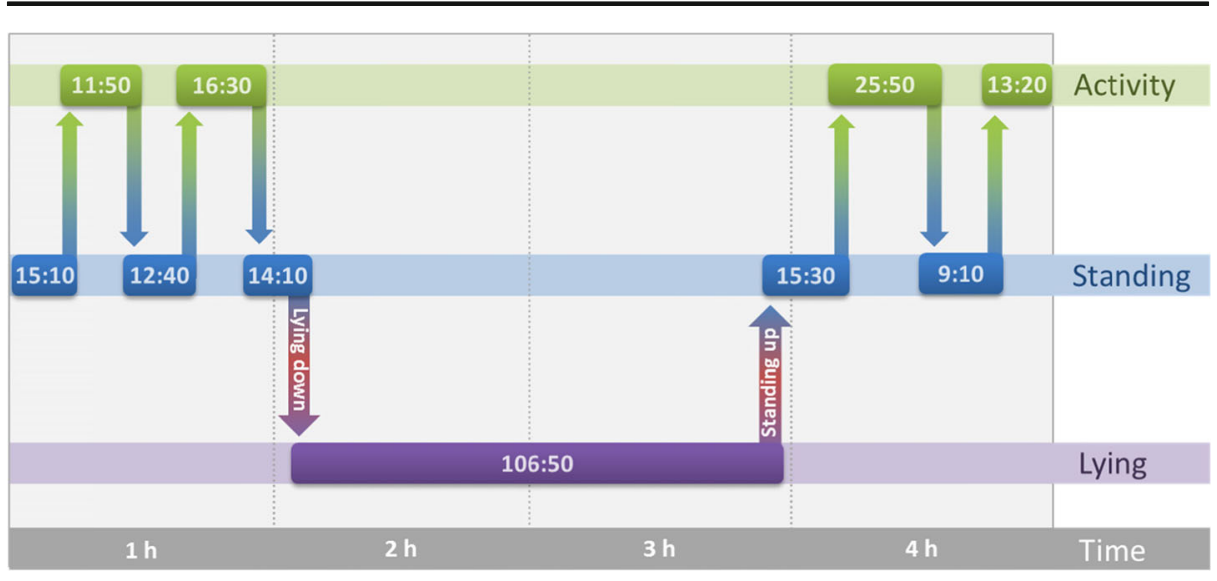

Fig. 2 "Digital Dairy Diary" of cow extracted by the combination of SVM and CRF based on a heart signal and the recorded trajectory. The pattern "activity" comprises the activity patterns "walking", "feeding", and "drinking"

CRFs, however, do rely on the Markov assumption that all necessary information on history is given in the current state. They do not provide a notion of time or duration, which, however, is crucial in the given scenario. Due to the involved strain cattle tend to remain in the state of lying for some time instead of standing up immediately. If this inertia is not modelled appropriately, this results in an erratic behavior of the model. Thus, CRFs had to be enhanced appropriately.

The application field of animal monitoring in barns requires an analysis method which is able to achieve the fine balance between a comprehensive data interpretation including spatial and stochastically prior knowledge and the extent of intervention by the sensor handling. These demands request a suitable combination and partial extension of state of the art methods. The underlying methodological concept is not limited to animal science but allows integrating various sensors and arbitrary distributions on state durations in various scenarios.

The contribution of this article is twofold. First, it introduces a novel sensor combination for an improved identification of activity states and locomotion patterns. In addition, SVM and CRF are combined [37] in a novel way. State transitions are modeled by a stochastic finitestate machine. This is realized by extending the concept of the CRF by an explicit notion of duration. This allows to take appropriate account of the perseverance in specific states. The enhanced CRF concept incorporates prior knowledge about state durations and prohibits activity transitions which are impossible due to spatial constraints.

The manuscript is structured in the following manner: Section 2 introduces the scenario and the applied sensor setup. Section 3 explains the analysis methodology including feature extraction, SVM and CRF. The extension of the CRF by duration-aware probabilities for activity transitions is shown. Section 4 provides the final results of the analysis, interim results and the Resting Pulse Rate (RPR) as a first application. The results are discussed Section in 5 and a conclusion about the method is provided in Section 6.

\section{Data acquisition}

The introduced combination of two sensor types deduces reliable information about the six behavior patterns described in Section 2.3. The used sensory systems are the local positioning 
system (LPM) from Abatec and heart rate sensors from Polar. The LPM provides information on position and locomotion and is described in Section 2.2. The heart rate sensor permits the derivation of information on local physical exertion and long-term stress load and is described in Section 2.3.

\subsection{Spatial context}

The study was conducted at the Frankenforst research station of the University of Bonn. A herd of 65 German Holstein Friesian cows is loose-housed in a two-row open free-stall barn with cubicles and concrete floor.

\subsection{Sensor for automatic recording of 3D location}

For the automatic recording of the cow position in real-time a local position measurement system (LPM, http://www.abatec-ag.com) based on runtime measurement using radio waves (around $5.8 \mathrm{GHz}$ ) and triangulation was used. The system was developed for sports like soccer games and modified in order to adapt to the conditions in a free stall barn. To cover the area and to minimize the shadings and reflections due to the barn equipment, 12 antennas (so called base stations) with known coordinates were mounted on the walls and under the roof. Furthermore one reference transponder with a defined position was located centrally. It sends a continuous signal to the base stations to synchronize them. In this experiment each cow wore a head-collar with a fixed transponder on the top. At pre-defined intervals one specific antenna (called the master base station) triggers the transponder to transmit a signal and hence, the position can be calculated.

Under ideal conditions, the system estimates the 3D position of a transponder with a frequency up to $1000 \mathrm{~Hz}$ with a standard deviation of $2 \mathrm{~cm}$ [22]. A cow barn provides suboptimal conditions due to the high amount of metal present, wet surfaces and the relatively small distances between the base stations. Therefore, antenna positions were optimized regarding $2 \mathrm{D}$ accuracy with the corresponding disadvantage that vertical distances could not be measured anymore (Fig. 1).

After the optimization, we evaluated the LPM system from Abatec AG by observing 10 control points for a week on a $24 \mathrm{~h}$ base. Every 10 seconds the measured coordinate was recorded. Huge differences were observed between the best point in the middle of the barn with a standard deviation of $9 \mathrm{~mm}$ (all points within a $1 \mathrm{~m}$ radius) and the worst point at the border of the barn with a standard deviation of $2.15 \mathrm{~m}$ (98\% percent of the data was outside the $1 \mathrm{~m}$ radius and $35 \%$ even outside the $3 \mathrm{~m}$ radius). The mean standard deviation over all points on all days was $19.2 \mathrm{~cm}$ as most of the control points were in the center of the barn. Summarizing, the accuracy depends strongly on the position and varies between precise and an accuracy that does not allow associating single lying boxes.

\subsection{Heart sensor}

For the measurement of the heart rate and the heart rate variability sensors from Polar Electro GmbH (Büttelborn, Germany) were used (Polar Equine RS800, Polar Equine RS800CX). The electrodes, integrated in a chest belt (Polar Equine WearLink® W.I.N.D. transmitter), measure electrical heart signals and transmit them wireless to a receiver with integrated data logger attached to a neck belt. An elastic surcingle and conductivity ultrasound gel was used to 
improve the contact of the sensors. The data was downloaded once a day to the corresponding software (Polar ProTrainer 5 Equine Edition - version 5.35.165) using an infrared interface.

\subsection{Experimental design}

The observations were taken at three periods at the same time of day: in the first two periods 4 cows were observed for at least $4 \mathrm{~h}$ and in the last period 5 cows were observed for at least $4 \mathrm{~h}$. Overall 43 valid time series for 12 different cows were recorded. The model was trained and evaluated using the data gathered in the two first observation periods. In these two periods more than $100 \mathrm{~h}$ with approximately 180,000 individual measurements of position and heart signal were recorded and annotated. In the last measurement period the local position measurement system was deactivated, because in this period the resting pulse rate should be tested for stability and relevance for parameters like pregnancy. Therefore the annotation was limited to the distinction between the states "lying" and "standing".

The direct observations of cow behavior were done with regard to five activity states (period I and II) defined in a protocol and used as labels for the training data. The used behavior states were "standing", "lying", "walking", "feeding" and "drinking". Synchronized digital stop-watches were used to measure the start time of each behavior state in seconds. The alteration of the behavior was used as start time for a new behavior category. The pattern "standing up" and "lying down" could not be annotated precisely enough. Hence, their point in time was deduced afterwards from the switch between "standing" and "lying" and their duration was set to 30 s (plausible estimation from own observations and in accordance with [38]).

\section{Identification of spatio-temporal activity sequences}

The data analysis which derives the sequence of activity states is divided in three steps (Fig. 3). In the first step, spatiotemporal features were extracted from filtered sensor data. In the second step, preliminary probabilities for activity states were derived by a multiclass Support Vector Machine (SVM [39]). In the third step, these probabilities were linked with each other and combined with contextual knowledge by a graphical model, more precisely by a Conditional Random Field (CRF [36]).

\subsection{Feature extraction}

The position and heart rate signals were observed by two independent sensors with different measurement rates. The LPM provides coordinates in around $2 \mathrm{~s}$ intervals and the heart parameters were derived once per second. Both signals were synchronized with an internet time server for the calculation of joint features (Table 1). Due to the higher measurement frequency of the heart rate sensor, the heart rate measurements were associated with the temporally closest position measurement. The result was a synchronized data set with the sampling frequency of the polar heart rate sensor.

As the signals of the introduced sensors were noisy and included outliers, e.g. measured positions outside the stall barn or heart frequencies over $250 \mathrm{bpm}$, the data sets were filtered before features for classification were extracted. A Gaussian and a nonlinear median filter were used for the data smoothing. For the smoothing of the positions, a median filter width of 30 


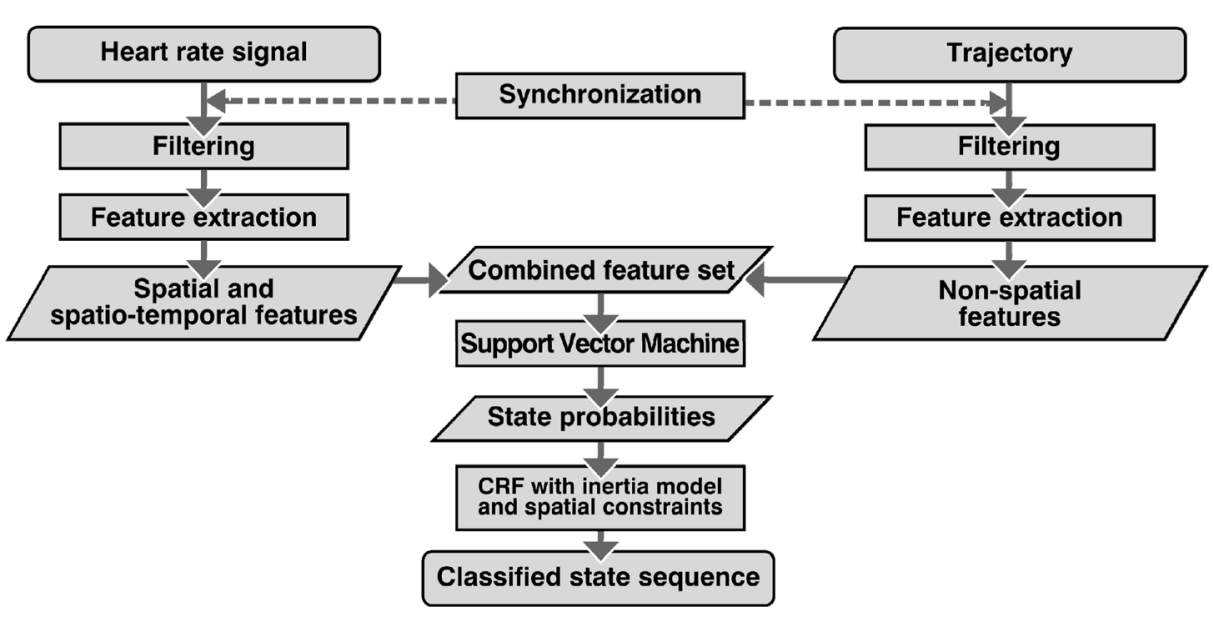

Fig. 3 Data analysis workflow including feature extraction, data fusion, SVM probabilities and final state sequence classification using the CRF model

observation periods and a Gaussian filter width of 40 periods were used. For the heart rate sensor both filters were used with filter width of 200 periods. The median filter applied to the position signal was triggered by the condition that the distance between the active and the preceding position was larger than $3 \mathrm{~m}$ which is a strong indication of an outlier.

Following, spatial and non-spatial features are extracted for each time step from the synchronized signals (Table 1). At the evaluation of the trajectories, absolute coordinates on its own does not contain much information as the different zones of the barn are close to each other. Prior knowledge about the structure of the stall barn has to be included in form of a map. The distances to the characteristic objects like feeders and water trough were used as features. Additionally the movement, specified by a significant change in position and the movement speed, and the information about the current barn zone (cubicle, walking alley and feeding alley) were included as features.

Table 1 List of all features extracted and used as input for the SVM classifier

\begin{tabular}{lll}
\hline Feature name & Type & Range of values \\
\hline Movement & Spatio-temporal & {$[0,1]$} \\
Barn zone & Spatial & {$[0,1,2]$} \\
Distance to feeding station & Spatial & $0-10 \mathrm{~m}$ \\
Distance to water station & Spatial & $0-12 \mathrm{~m}$ \\
Distance walked in 10s & Spatio-temporal & $0-8 \mathrm{~m}$ \\
Smoothed distance walked & Spatio-temporal & $0-6 \mathrm{~m}$ \\
Heart rate & non-spatial & $40-120 \mathrm{bpm}$ \\
RMSSD & non-spatial & $5-330 \mathrm{~ms}$ \\
Recurrence & non-spatial & $0.001-0.2$ \\
Determinism & non-spatial & $0.8-1$ \\
Entropy & non-spatial & $1.5-4$ \\
MaxLine & non-spatial & $10-250$ \\
Trend & non-spatial & $-70-70$ \\
\hline
\end{tabular}


RMSSD ("root mean square of successive differences") was used as HRV parameter [25] and was added together with the HR to the feature set. Additional features were gained from Recurrence Quantitative Analysis (RQA; [27]), namely Recurrence, Determinism, Entropy, MaxLine and Trend. These features were successfully applied by Mohr et al. [28] for the stress assessment at cows. Thus, the same parameter settings were used and the features were calculated by the CRP Toolbox 3.19 from the Potsdam Institute for Climate Impact Research [40].

Overall 13 features (Table 1) combined in a feature vector. A feature vector is provided each second and used as input for the SVM.

\subsection{Machine Learning method for spatio-temporal data analysis}

\subsubsection{Support Vector machine (SVM)}

SVMs are a state of the art method for supervised classification and provide linear and nonlinear discrimination functions [39, 41]. As well as their ability to provide non-linear discriminators their specific advantage is that they generalize well from the training data to new unobserved cases. SVMs contain "Kernel” functions specifying distances metrics in the space of observations [42]. The most common kernels are radial basis functions (rbf) which were also used in our study.

The model of a SVM consists of a subset of weighted data instances - so called support vectors (SV). They define a hyperplane separating two classes whereas its position is defined by the principle of a maximum margin. In the separable case the margin is intuitively conceivable as the smallest distance between the hyperplane and a training data point. This principle of maximum margin facilitates the learning of models with excellent generalization performance and robustness.

In recent years SVMs have been successfully applied in various fields, e.g. remote sensing and genetics. This method was used for tasks from precision agriculture [43] ranging from the detection of meat and bone meal [44] to determination of biotic stress in agrarian plants [45]. The application of SVMs for activity pattern prediction of dairy cows with accelerometers was examined by Martiskainen et al. [18].

The accuracy and performance of the SVM depends on the selection of a suitable model. The model type is controlled by the chosen kernel function, the corresponding parameters and the penalty weight $C$ [41]. For the given task, the rbf kernel achieves the best result due to the nonlinear class boundaries included in the data. The combined optimization of the kernel width $\sigma$ and the penalty weight $\mathrm{C}$ is done by a $2 \mathrm{~d}$ grid optimization $\left(\mathrm{C}=2^{-5} \ldots 2^{10}, \sigma=2^{-10} \ldots 2^{5}\right)$ in conjunction with cross validation.

The classification and probability estimation was done by an rbf-SVM (LIBSVM, version 3.11 [46]). As input data, the features shown in Table 1 were normalized and used. For the application of the graphical model in the next section the presented method demands on class probabilities for every observation. These probabilities were estimated following Lin et al. [47] by scaling the decision values, meaning the distance to the discriminating function. The scaling is a mapping of the decision values $[-\infty,+\infty]$ to posterior probabilities $[0,1]$ for every class. The derivation of the class probabilities of a complete time series was conducted as follows: first, a SVM model was derived from the remaining time series (including cross-validation for parameter optimization). Following, for each time step in the current time series the probabilities for each class were predicted. This process was repeated for each available time series. In 
this way it was guaranteed that only general patterns included in every time series are used for prediction and that no over-fitting effects are included in the model.

The result of this analysis step is a probability for each activity state in each observation period for all observed time series.

\subsubsection{Conditional random field}

Graphical Models [48] - with CRFs as one particular instance - are an established method to represent prior knowledge in models. The data and the unknown conclusions are represented by nodes linked by edges denoting possible dependencies. This general approach has been adapted to several kinds of graphical models differentiated in graphical models with directed and undirected edges.

Bayesian networks use directed edges to represent the conditional influence of one node on another [49]. Markov random fields (MRFs) use undirected nodes to define the relation between the random variables that follow the Markov property. The Markov property states that a variable is conditionally independent of all other variables if its neighbor nodes are observed. CRF includes edges of both types shown in Fig. 4 [36]. Attached to the links are distributions which describe the relation of the linked nodes and are represented by distribution parameters.

The developed CRF model is composed of two node types, observations nodes and unobserved label nodes, and two edge types: The undirected edge between the observations and the hidden behavior patterns and the directed edge between succeeding behavior pattern nodes (Fig. 3). The observation nodes and the undirected edges were realized by an SVM model [37]. The parameters of corresponding probability functions were optimized in SVM optimization.

For our specific application there is still the problem of the Markov assumption included in the inference step. The Markov assumption states that all the knowledge about the preceding sequence of activities is representable by the current state. In fact, if a cow is "lying", there is a high probability that the subsequent state is again "lying", a much lower probability that it is "standing up", but a probability of zero that it is "standing". The latter is caused by the constraint that the state "standing up" has to be in between. The high probability for remaining in "lying" is caused by an inherent inertia: after "lying down" the cow will rest for a while and - as a rule - not "stand up" again immediately. This inertia cannot be modeled neither by HMMs nor CRFs as they are. For this reason we have adapted the CRF model by incorporating a time counter. We record the number of time steps in a given state and adapt the a priori

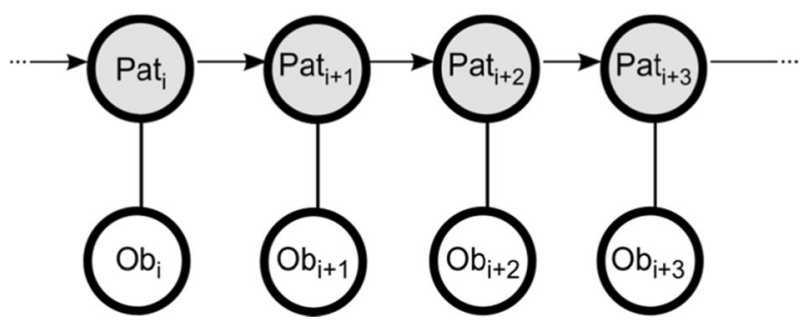

Fig. 4 Graphical representation of a chain structured Conditional Random Field (CRF) connecting the locally measured observations " $\mathrm{Ob}_{\mathrm{i}}$ " with the sequence of hidden activity patterns "Pat ${ }_{i}$ ". The undirected links between the observation nodes and the activity nodes are realized by the SVM probabilities and the directed links between the activity nodes by a duration-aware transition matrix 
probability for a state transition by the duration of the current state. The Viterbi algorithm [50] inferring the most probable label sequence has been modified appropriately (Alg. 1). It was enhanced to estimate the most probable duration $d_{i}$ of the active state $i$. This duration is used to adapt the transition probabilities and represent the inertia of the cow (Fig. 5). The original Viterbi-algorithm relies on constant transition probabilities leading to a geometric cumulative distribution function (CDF).

The Viterbi algorithm was enhanced by a tracing step executed for every node, in which the most probable sequence based on the preceding observations is determined (Algorithm 1 [51]). From this label sequence the duration $d_{i}$ was determined as the number of steps since the last state change:

$$
d_{i}=i^{-}\left(\max k \mid \text { state }_{k} \neq \text { state }_{i} ; k=i-1 \cdots 0\right)
$$

The local tracing can be truncated if a state change is reached or alternatively congruence with the preceding local tracing is found. The extracted duration is used for the adaption of the transition matrix $a$ by multiplying the transition probabilities with the value of a cumulative distribution function (CDF) at position $d_{i}$ :

$$
a_{d u r}[s, i]=C D F\left(d_{i}\right) * a[s, i]
$$

The application for activity pattern recognition requires the definition of functions of $d_{i}$ for the transition matrix. Consequently two different kinds of transition functions are used, one for fast state transitions and one for a longer persistence in a state. A semiMarkov process is shown in Fig. 4, where possible transitions are shown by an arrow and the different types of states are marked by node and edge color. An example for a short persistence in a state is "drinking" (orange) and for a long persistence is "lying" (green).

A rough estimation of the parameters describing these duration distributions (CDFs) was sufficient because of the high variability in the individual state durations in the data. For the short persistence like "feeding", "drinking" and "walking" a linear model (Eq. 3) is used and for the longer persistence like "lying" and "standing" a sigmoid function (Eq. 4) modeling the transition function is used. It has the ability to suppress extremely short state sequences and is constant at a higher $d$. Also the linear model tends to suppress state durations of few seconds because these seem implausible but in contrast to the sigmoid model, the probability for transition at higher duration times has clearly increased and is constantly growing. This transition function reduces the probability for a longer persistence in a short state.

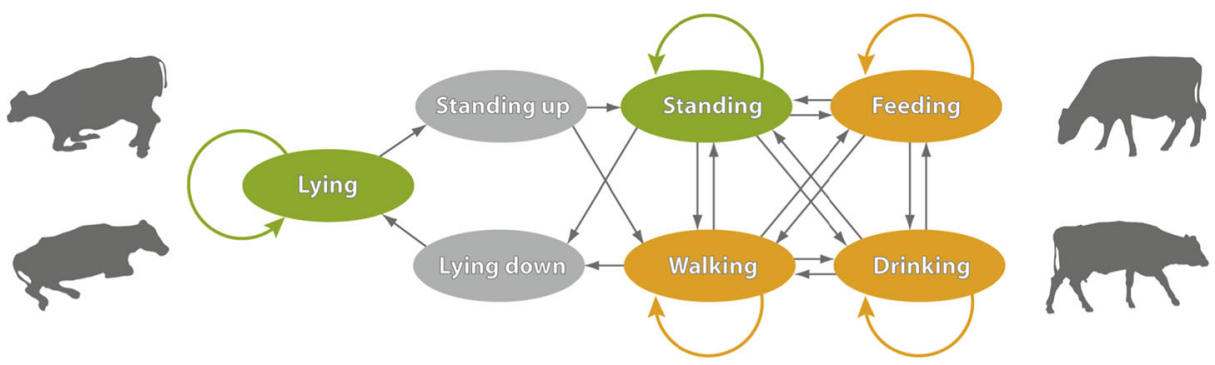

Fig. 5 Visualization of the activity states and the possible state transitions. The colors distinguish the short (orange) and long (green) duration states from the inter-states (grey). The constraints and different state durations are realized by a sparse and duration aware transition matrix 


$$
\begin{gathered}
C D F_{\text {short }}(d)=500 * d * 10^{-6} \\
C D F_{\text {long }}(d)=\frac{1}{1+e^{-0.03 *(10 * d-500)}}
\end{gathered}
$$

Another advantage of using a transition matrix is the possibility to introduce contextual knowledge about fundamental movement constraints of the animal in the stall environment. Therefore it is possible to avoid specific transitions in the resulting sequence e.g. from "lying" to "standing". In this manner, it can be forced that the pattern "standing up" has to occur in the meantime (Fig. 4). Hence the generated time series of labels is in every case valid from the viewpoint of physical constraints of the cattle in the stall.

Alg 1. Adapted Viterbi-algorithm for determination of the most probable sequence considering the duration dependent transition matrix and the local determination of the duration of the current state.

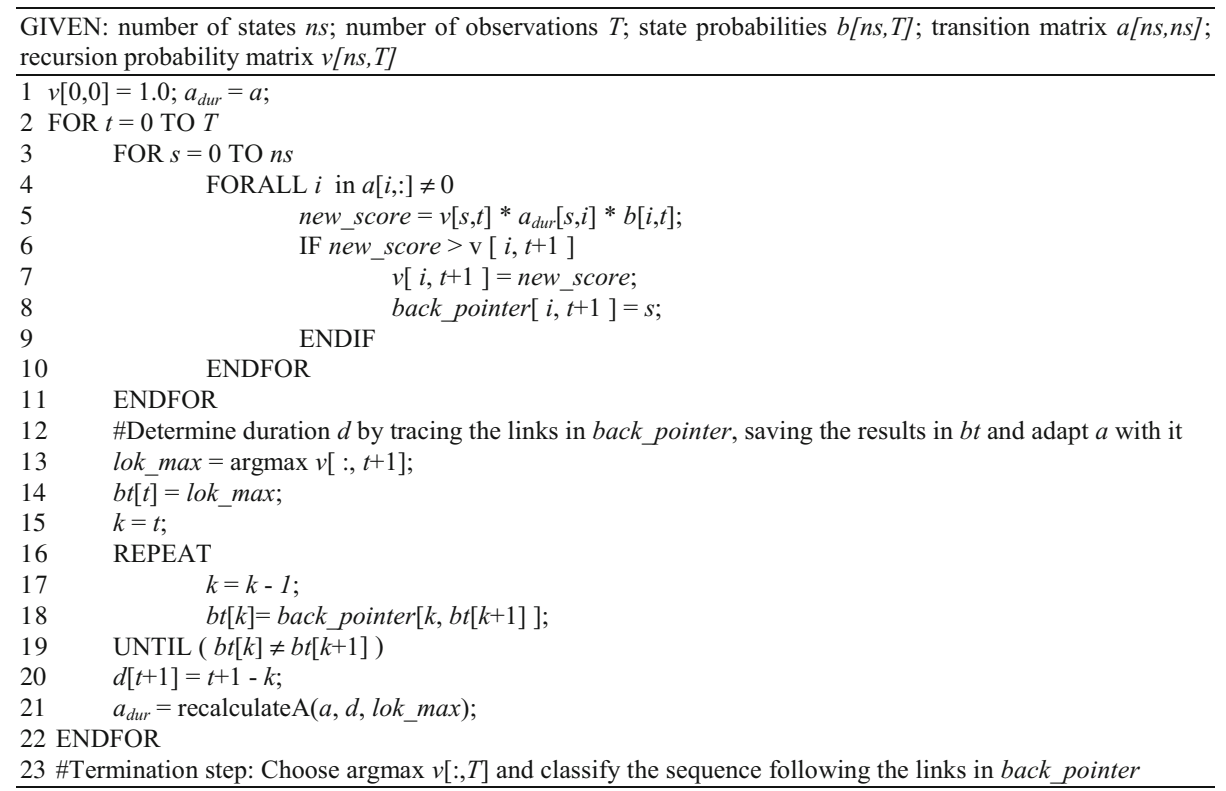

These extensions are induced in the Viterbi algorithm as shown in Alg. 1. The original algorithm covers lines 1 to 11 and lines 22 and 23. The lines 12 to 20 contain the determination of most probable duration $d$ (lines 16 to 20) of the most probable current state lok_max (lines 13 and 14). The transition matrix $a_{d u r}$ is then adapted the determined duration in line 21 . These adapted transition probabilities are used in the next iteration of the Viterbi algorithm (lines 3 to 11).

The resulting CRF model contains a SVM classification [37] for feature interpretation and models the transition probabilities between neighbored state nodes by a transition matrix depending on the duration of the current state. 


\section{Results and discussion}

The validation of the classification model was done by cross validation on the described data set. This was done, not on the level of single observations, but on time series level. This approach excludes the whole time series to classify from the training data that is extracted from the remaining time series. Otherwise, the strong similarity between neighboring points would prevent an unbiased evaluation of the result quality.

For building a "Digital Dairy Diary", the most probable sequence of labels with the given observations and considering the physical constraints has to be stored. One sample time series is presented in Fig. 6 showing the accuracy of the classification by the SVM and its improvement by the additional use of the graphical model. SVMs as state-of-the-art classifier achieve an overall accuracy of $79.01 \%$ (average over all states) for the classification of activity pattern on its own. Consequently, it outperforms the widespread Naive Bayes approach $(\mathrm{Acc}=56.33 \%)$ and is comparable to the Random Forest classifier $(\mathrm{Acc}=78.01 \%)$.

The SVM results were improved by the Graphical Model (Fig. 6 and Table 2). The used CRF enables the introduction of sequence information and can be adapted to the task of activity pattern determination by including time dependent transition functions. Implausible short sequences caused by outliers would interrupt these sequences and reduce the accuracy of the extracted parameters. This is taken into account by introducing the semi-Markov process. This additional duration dependency has drastically reduced the number of state changes. This focus is considered by utilizing additional quality measurements. Besides the accuracy in percent the "Longest Common Subsequence" (LCS) and all "Common Subsequences" (CS) demonstrate the improved result quality of the CRF.

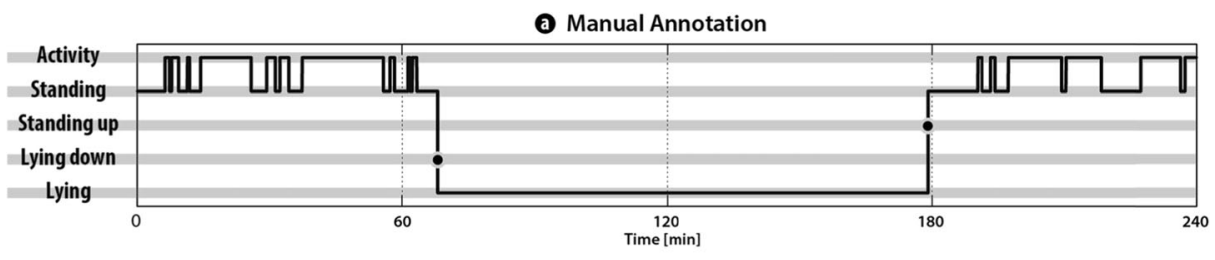

D Classification result of the SVM (Accuracy $87.3 \%$ / \# of state changes: 258 )

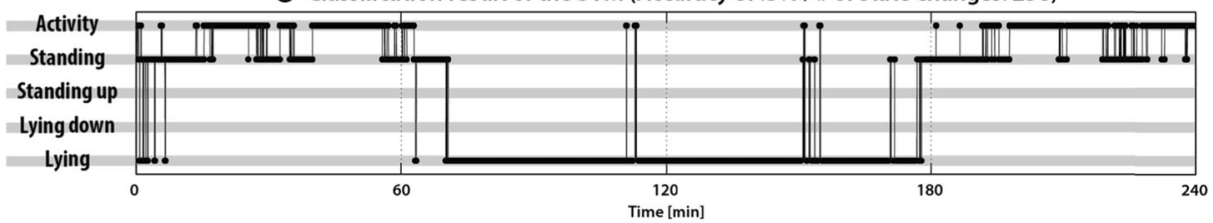

C Classification result of the SVM + CRF (Accuracy $88.2 \%$ / \# of state changes: 9)

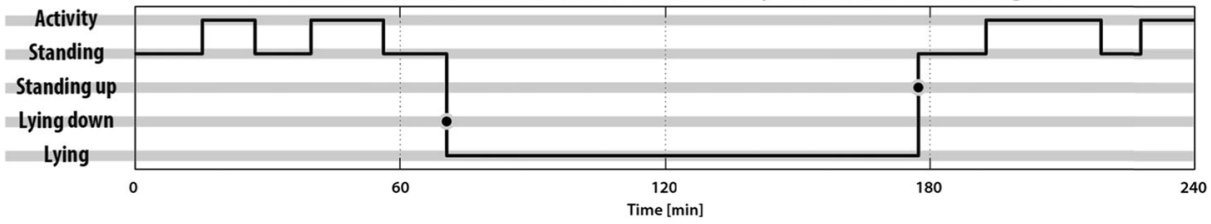

Fig. 6 Prediction of activity states showing the different characteristics of the SVM classification result and the result of the graphical model. From top to bottom: a the manual annotation, b prediction of the SVM and c prediction of the CRF. The provided prediction accuracies are related to the shown time series. The pattern "activity" comprises the active pattern "walking", "feeding" and "drinking" 
Table 2 Comparison of classification results of the SVM and the CRF

\begin{tabular}{llr}
\hline Evaluation Parameter & SVM & CRF \\
\hline Mean \# state changes & 328 & 6 \\
Mean length of LCS & 3481 & 6207 \\
Mean length of all CS & 107 & 1271 \\
Accuracy "Lying" & $84 \%$ & $86 \%$ \\
Accuracy "Standing-Meta" & $81 \%$ & $89 \%$ \\
\hline
\end{tabular}

The upper three evaluation parameters deal with the interpretability and sequence accuracy (Longest Common Subsequence (LCS) and Common Subsequences (CS)). The lower two parameters provide the absolute accuracy in distinguishing lying and standing. The meta states "Lying" and "Standing-Meta" comprise all lying and standing states, respectively

The improvement is based on the smoothing mechanism of the CRF which is controlled by defined probability distribution functions (Fig. 5 and Table 2). In this way, the model is able to handle the high noise level with regard to specific states and underlying movement models.

The proposed method determines the activity patterns in a physically plausible order whilst also creating an activity state sequence that is valid, given the boundary conditions of the barn and the cattle physiology. In the cases of "standing up" and "lying down" in Fig. 5 the point in time was correctly determined according to the state transition model in Fig. 4. This effect is forced by the model due to the included contextual knowledge.

The general trends in all analyzed time series correspond to the properties of the visualized time series in Fig. 5. The mean accuracies of different states and model quality parameters are shown in Table 2, confirming the desired effects. The model is able to determine reliably between "standing" and "lying" - although there is no height information from the positioning system. The resulting accuracies of the individual states reveal the difficulties of the monitoring task. Some states are very similar, e.g. feeding, standing and walking causing many misclassifications and "drinking" as extremely rare state in the data set are never predicted (Table 3). The low accuracies of "walking" and "standing" are related to varying granularities of annotation. Typically, the cow switches multiple times between "standing", "walking" and "feeding" during a visit at the feeding alley. Multiple feeding stations are visited and in between the states "standing" and "walking" occur (Fig. 1). Therefore, the temporal accuracy and the level of summarization of the annotation have highest impact on the validation result. The model catches these sequences by the most probable state series enabling a high level of interpretability (Fig. 5).

Table 3 Accuracy partition of the "standing" states

\begin{tabular}{llr}
\hline State accuracy & SVM & CRF \\
\hline Accuracy "Lying" & $84 \%$ & $86 \%$ \\
Accuracy "Standing-Meta" & $81 \%$ & $89 \%$ \\
Accuracy "Walking" & $16 \%$ & $18 \%$ \\
Accuracy "Feeding" & $76 \%$ & $82 \%$ \\
Accuracy "Standing" & $41 \%$ & $42 \%$ \\
Accuracy "Drinking" & $0 \%$ & $0 \%$ \\
\hline
\end{tabular}

The low accuracies in "walking" and "standing" are related to the high level of similarity and the temporal resolution. The incapability to detect the state "drinking" is related to the rare occurrence of this state in the data 
Overall, the reached accuracies should be evaluated in the light of high sensor noise, especially in the case of positioning systems. Standard deviations of more than $4 \mathrm{~m}$ prevent a reliable assignment to a box and essentially complicate a state determination. The reliable determination of "lying" and "standing" states shows the potential of the proposed method even under roughest conditions.

The sequence of activity patterns (especially the count and length of the resting phases during a day) provides useful information about the physiological condition of individual cattle. Table 3 shows the improvement of the classification by the CRF. Slight improvements in all accuracy are accompanied by significantly improved opportunity to interpret automatically the classification results (shown by LCS and mean CS). Long sequences like "lying" are classified without single errors, which enable the robust extraction of parameters like "longest lying sequence" or "count of standing up sequences". The developed transition model (Fig. 4) supports the robust determination of long lying periods by the explicit modeling of "standing up" and "lying down". The difference of slow walking and standing is, from a technical point of view, imprecise and, therefore, the annotation quality varies strongly. The shown smoothed state summary was desired and, as the longer states were in focus, does not reduce the value of the results for application.

The presented method overcomes the limitation of absent height information which is essential for distinguishing "standing" and "lying". The combination of two sensors (heart rate and location sensor) and the analysis of their signals with a combination of two classifiers improve significantly the classification results.

\subsection{Automated derivation of resting pulse rate}

During the study it became obvious that the variance of the HR while lying (Resting Pulse Rate, RPR) of an individual cow was distinctly lower than the variance within the whole herd. To make this explicit the mean and the standard deviation of the median heart rate while lying on the three observation days per individual animal was calculated (Table 4).

Table 4 Cattle specific Mean Resting Pulse Rate (RPR) and its standard deviation derived from at least three plausible observations

\begin{tabular}{lll}
\hline Days of pregnancy elapsed & $\begin{array}{l}\text { Resting Pulse } \\
\text { Rate [bpm] }\end{array}$ & $\begin{array}{l}\text { Standard } \\
\text { deviation [bpm] }\end{array}$ \\
\hline 38 & 64.9 & 1.37 \\
99 & 65.0 & 3.66 \\
120 & 70.7 & 1.57 \\
162 & 68.9 & 2.29 \\
207 & 69.9 & 2.52 \\
211 & 75.4 & 0.29 \\
214 & 75.0 & 2.81 \\
218 & 81.0 & 1.28 \\
- & 73.5 & 2.06 \\
- & 65.7 & 1.11 \\
\hline
\end{tabular}

The derived lying sequences allows to calculate automatically the RPR and to verify its significant correlation to the day of pregnancy 
The mean resting pulse of the ten individual animals varied from $64.9 \mathrm{bpm}$ to $81.0 \mathrm{bpm}$. The mean standard deviation of an individual cow was $1.96 \mathrm{bpm}$ (within the range of $0.29 \mathrm{bpm}$ to $3.66 \mathrm{bpm}$ ). Furthermore it was noticed that the average heart rate per cow dependent significantly of the respective pregnancy stage ( $p=0.049$; by Kruskal-Wallis H Test): In the first 100 days on average $65.0 \mathrm{bpm}(n=2)$, for 101200 days of pregnancy on average $69.8 \mathrm{bpm}(n=2)$ and in the last pregnancy period starting at day 200 on average $75.3 \mathrm{bpm}(n=4)$ was recorded. The cow no. 44 with the longest pregnancy duration (day 218) had the highest resting pulse rate of $81.0 \mathrm{bpm}$ (Fig. 7).

\section{Conclusion and perspectives}

We presented an approach for the classification of spatio-temporal activity states based on multiple sensors. We applied the method to a data set of dairy cows but it is generalizable to the detection of activity sequences based on heterogeneous, spatial and non-spatial sensor signals. We have shown that the method utilizes the non-spatial signals to bridge the activity states without spatial effects like the distinction of standing and lying without height information.

We have shown that the proposed method is able to improve the determination of activities by considering contextual knowledge about distribution information and constraints. The model design supports the inclusion of contextual knowledge by considering time and space dependent activity properties by the adaption of the transition probabilities. Furthermore, the definition of an explicit movement model like at a Kalman filter is not required to combine spatial and non-spatial features. The machine learning algorithms derive the relations between the features and the activity states directly from the data. In this way, the result quality benefits particularly from the time series characteristic of the data. Additional prior knowledge about the inertia of the moving object improves the result quality further.

An advantage of the combination of two discriminative classification methods is that the modeling of the unknown statistical data distribution that causes a decreasing model quality (if wrong assumptions were made), can be neglected. Another advantage of the presented model with two stages is the improved interpretability of the result. The interim result of the SVM can

Fig. 7 Visualization of the relation between day of pregnancy and automatically derived RPR (Table 4). A significant correlation was verified

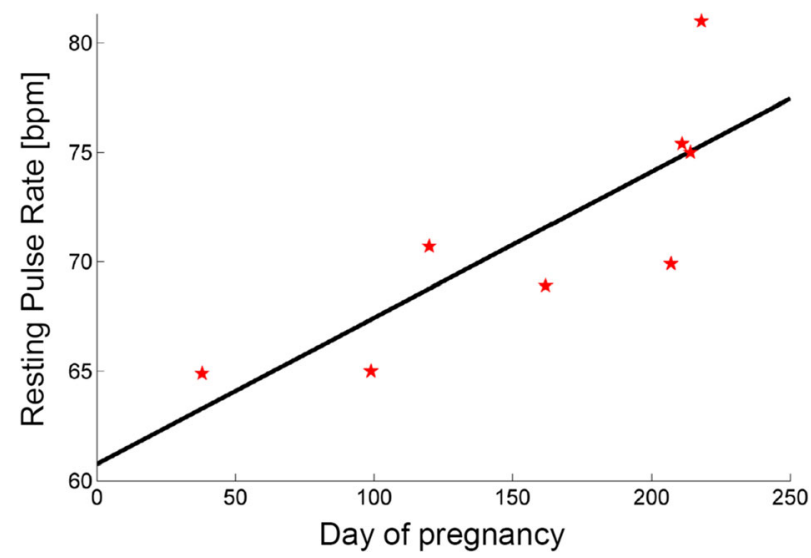


be visualized (Fig. 6) and each step optimized on its own. The model is suited to determine the activity pattern by combining the position and additional signal source in various scenarios. This task is highly relevant, e.g. for the increasing requirements for monitoring high performance dairy cows.

The field of application for such a system will be strongly related to the public debate on animal welfare and behavior science. With minimal interference, extensive experiments on the response of cattle to different housing conditions and cowshed layouts can derive wellbeing standards based on objective, reproducible and automated observations.

Furthermore the final result enables a clearer view regarding the individual properties and supports an automatic interpretation as the preliminary task for a "Digital Dairy Diary" (Fig. 2). On a $24 / 7$ basis, recorded activity profiles can then be used for reconstructing the case history of disorders. The definition of "normal behavior" derived from a wider data base will enable the detection of deviations leading to a root cause analysis.

Moreover, it enables an automatic evaluation of the heart rate signal by detecting comparable situations on different days. An example of such application is the automatic determination of a representative pulse rate while "lying" (so called resting pulse rate) without additional visual annotation. First analyses show a significant correlation between the resting pulse rate and physiological states. For this reason it is also used in human medicine - the heart rate measurement while in the resting phase (the resting pulse rate) is used for clinical assessment purposes [52]. For these reasons, the individual animal heart rates on the days of measurement stay constant and are thus significant data sources (Table 4). In human physiology it is known that the heart rate is affected by certain underlying conditions, regardless of internal and external stressors; these conditions include sex, age and pregnancy $[52,53]$. In dairy cows it has been shown that the heart rate increases significantly with positive variations in body weight and as the day goes forward [26]. From our own research we are able to prove significantly that with the increasing days of gestation of the pregnant dairy cow, the heart rate increases. This relation is already known at human physiology [53], but has not yet been considered in studies of dairy cows. Further studies with a larger number of animals should be undertaken so that this presumption can be examined statistically in depth.

The sensor selection reflects a compromise between the amount of information and the degree of interference with normal cattle behavior. Great importance has been attached to the simultaneous sensing of non-redundant signals, the inner (heart rate sensor) and outer condition (position sensor). The two sensor types complement each other and give an integrated view of the individual characteristics of the cattle. Further studies may take effort of this potential.

The integration of additional sensors like rumination sensors or pedometers in this model is possible and straight forward by expanding the feature vector. Combination with accelerometers would probably improve the accuracy of positions derived from the LPM-system by reducing the local noise and increasing the short-time precision. With new information the classification quality would increase. However, additional sensors require additional synchronization and increase the effort for continuous monitoring.

Acknowledgements This research was conducted in the Center of Integrated Dairy Research (CIDRe), University of Bonn (Bonn, Germany). 
Open Access This article is distributed under the terms of the Creative Commons Attribution 4.0 International License (http://creativecommons.org/licenses/by/4.0/), which permits unrestricted use, distribution, and reproduction in any medium, provided you give appropriate credit to the original author(s) and the source, provide a link to the Creative Commons license, and indicate if changes were made.

\section{References}

1. Andersson M, Gudmundsson J, Laube P et al (2008) Reporting leaders and followers among trajectories of moving point objects. Geoinformatica 12(4):497-528

2. Sakr M, Güting RH (2011) Spatiotemporal pattern queries. Geoinformatica 15(3):497-540

3. Parent C, Pelekis N, Theodoridis Y et al (2013) Semantic trajectories modeling and analysis. ACM Comput Surv 45(4):1-32. doi:10.1145/2501654.2501656

4. Herrera JC, Work DB, Herring R et al (2010) Evaluation of traffic data obtained via GPS-enabled mobile phones: the mobile century field experiment. Transport Res C-Emer 18(4):568-583

5. Höferlin M, Höferlin B, Weiskopf D et al (2011) Uncertainty-aware video visual analytics of tracked moving objects. JOSIS. doi:10.5311/JOSIS.2010.2.1

6. Leser R, Baca A, Ogris G (2011) Local positioning systems in (game) sports. Sensors 11(10):9778-9797

7. Anderson D, Estell R, Cibils A (2013) Spatiotemporal cattle data - a plea for protocol standardization. Positioning 4(1):115-136

8. Laube P, Imfeld S, Weibel R (2005) Discovering relative motion patterns in groups of moving point objects. Int J Geogr Inf Sci 19(6):639-668

9. von Borell E, Langbein J, Després G et al (2007) Heart rate variability as a measure of autonomic regulation of cardiac activity for assessing stress and welfare in farm animals - a review. Physiol Behav 92(3):293-316

10. Liao L, Fox D, Kautz H (2007) Extracting places and activities from gps traces using hierarchical conditional random fields. Int J Robto Res 26(1):119-134

11. Shamoun-Baranes J, van Loon EE, Purves RS et al (2012) Analysis and visualization of animal movement. Biol Lett 8(1):6-9. doi:10.1098/rsbl.2011.0764

12. Laube P, Purves R (2011) How fast is a cow? cross-scale analysis of movement data. Trans GIS 15(3):401418

13. Wang H, Kläser A, Schmid C et al (2013) Dense trajectories and motion boundary descriptors for action recognition. Int J Comput Vis 103(1):60-79. doi:10.1007/s11263-012-0594-8

14. Mirat O, Sternberg JR, Severi KE et al (2013) ZebraZoom: an automated program for high-throughput behavioral analysis and categorization. Front Neural Circuits. doi:10.3389/fncir.2013.00107

15. Yang B, Nevatia R (2014) Multi-Target tracking by online learning a CRF model of appearance and motion patterns. Int J Comput Vis 107(2):203-217. doi:10.1007/s11263-013-0666-4

16. O'Driscoll K, Boyle L, Hanlon A (2008) A brief note on the validation of a system for recording lying behaviour in dairy cows. Appl Anim Behav Sci 111(1):195-200

17. Cornou C, Lundbye-Christensen S (2008) Classifying sows' activity types from acceleration patterns: an application of the multi-process Kalman filter. Appl Anim Behav Sci 111(3):262-273

18. Martiskainen P, Järvinen M, Skön J et al (2009) Cow behaviour pattern recognition using a threedimensional accelerometer and support vector machines. Appl Anim Behav Sci 119(1-2):32-38

19. Schlecht E, Hülsebusch C, Mahler F et al (2004) The use of differentially corrected global positioning system to monitor activities of cattle at pasture. Appl Anim Behav Sci 85(3):185-202

20. Wark T, Corke P, Sikka P et al (2007) Transforming agriculture through pervasive wireless sensor networks. Pervasive Comput 6(2):50-57

21. Huhtala A, Suhonen K, Mäkelä P et al (2007) Evaluation of instrumentation for cow positioning and tracking indoors. Biosyst Eng 96(3):399-405

22. Pourvoyeur K, Stelzer A, Gassenbauer G (2006) The Local Position Measurement System LPM used for Cow Tracking. In: Multisensor Fusion and Integration for Intelligent Systems, 2006 I.E. International Conference on, pp 536-540

23. Gygax L, Neisen G, Bollhalder H (2007) Accuracy and validation of a radar-based automatic local position measurement system for tracking dairy cows in free-stall barns. Comput Electron Agr 56(1):23-33

24. Neisen G, Wechsler B, Gygax L (2009) Effects of the introduction of single heifers or pairs of heifers into dairy-cow herds on the temporal and spatial associations of heifers and cows. Appl Anim Behav Sci 119(3): $127-136$ 
25. Langbein J, Nürnberg G, Manteuffel G (2004) Visual discrimination learning in dwarf goats and associated changes in heart rate and heart rate variability. Physiol Behav 82(4):601-609

26. Hagen K, Langbein J, Schmied C et al (2005) Heart rate variability in dairy cows-influences of breed and milking system. Physiol Behav 85(2):195-204

27. Webber JCL, Zbilut JP, van MA. Riley OG (2005) Recurrence quantification analysis of nonlinear dynamical systems. Tutorials in contemporary nonlinear methods for the behavioral sciences

28. Mohr E, Langbein J, Nürnberg G (2002) Heart rate variability: a noninvasive approach to measure stress in calves and cows. Physiol Behav 75(1):251-259

29. Marques A, Silverman MN, Sternberg EM (2010) Evaluation of stress systems by applying noninvasive methodologies: measurements of neuroimmune biomarkers in the sweat, heart rate variability and salivary cortisol. Neuroimmunomodulation 17(3):205-208

30. Nguyen N, Guo Y (2007) Comparisons of sequence labeling algorithms and extensions. In: Proceedings of the 24th international conference on Machine learning, pp 681-688

31. Shendure J, Ji H (2008) Next-generation DNA sequencing. Nat Biotechnol 26(10):1135-1145

32. van Halteren H, Daelemans W, Zavrel J (2001) Improving accuracy in word class tagging through the combination of machine learning systems. Computational linguistics 27(2):199-229

33. Zhang Y (2008) Progress and challenges in protein structure prediction. Curr Opin Struct Biol 18(3):342348

34. Koller D, Friedman N (2009) Probabilistic graphical models: principles and techniques. The MIT Press, Cambridge, Massachusetts

35. Rabiner L (1989) A tutorial on hidden Markov models and selected applications in speech recognition. Proc IEEE 77(2):257-286

36. Lafferty JD, McCallum A, Pereira FCN (2001) Conditional random fields: probabilistic models for segmenting and labeling sequence data. In: Proceedings of the eighteenth international conference on machine learning. Morgan Kaufmann Publishers Inc, San Francisco, pp 282-289

37. Hoefel G, Elkan C (2008) Learning a two-stage SVM/CRF sequence classifier. In: Proceedings of the 17th ACM conference on information and knowledge management. ACM, New York, pp 271-278

38. Österman S, Redbo I (2001) Effects of milking frequency on lying down and getting up behaviour in dairy cows. Appl Anim Behav Sci 70(3):167-176

39. Cortes C, Vapnik V (1995) Support-vector networks. Mach Learn 20(3):273-297

40. Marwan N, Wessel N, Meyerfeldt U et al (2002) Recurrence plot based measures of complexity and their application to heart rate variability data. Phys Rev E Stat Nonlin Soft Matter Phys 66(2): 26702

41. Schölkopf B, Smola A (2002) Learning with kernels: support vector machines, regularization, optimization, and beyond. The MIT Press, Cambridge

42. Boser B, Guyon I, Vapnik V (1992) A training algorithm for optimal margin classifiers. In: Proceedings of the fifth annual workshop on Computational learning theory, pp 144-152

43. Huang Y, Lan Y, Thomson S et al (2010) Development of soft computing and applications in agricultural and biological engineering. Comput Electron Agr 71(2):107-127

44. Mucherino A, Papajorgji P, Pardalos P (2009) Data mining in agriculture. Springer Optimization and its Application, vol 34. Springer Verlag

45. Rumpf T, Mahlein A, Steiner U et al (2010) Early detection and classification of plant diseases with support vector machines based on hyperspectral reflectance. Comput Electron Agr 74(1):91-99

46. Chang C, Lin C (2011) LIBSVM: a library for support vector machines. ACM Trans Intell Syst Technol 2(3):27

47. Lin H, Lin C, Weng R (2007) A note on Platt's probabilistic outputs for support vector machines. Mach Learn 68(3):267-276

48. Koller D, Friedman N (2009) Probabilistic graphical models principles and techniques. Adaptive computation and machine learning. MIT Press, Cambridge

49. Russell SJ, Norvig P (2003) Artificial intelligence: a modern approach prentice hall series in artificial intelligence, 2nd edn. Prentice-Hall, Upper Saddle River

50. Viterbi A (1967) Error bounds for convolutional codes and an asymptotically optimum decoding algorithm. IEEE Trans Inf Theory 13(2):260-269

51. O'Connell J, Tgersen F, Friggens N et al (2010) Combining cattle activity and progesterone measurements using hidden semi-Markov models. J Agric Biol Envir S 16(1):1-16

52. Ostchega Y, Porter KS, Hughes J et al (2011) Resting pulse rate reference data for children, adolescents, and adults: United States, 1999-2008. Natl Vital Stat Rep 41:1-16

53. Peña MA, Echeverrínez A, Vargas-García C et al (2011) Short-term heart rate dynamics of pregnant women. Auton Neurosci 159(1-2):117-122 


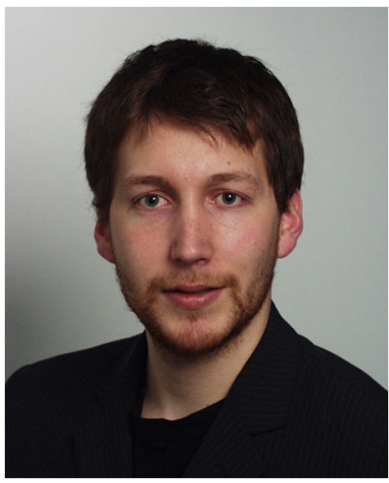

Jan Behmann is a PhD student at the Institute of Geodesy and Geoinformation in the department of Geoinformation at the University of Bonn. He has studied Geodesy and Geoinformation and completed his Bachelor in 2009 and his Master in 2011. Afterwards he has affiliated his actual position. His research deals with the analysis of complex and heterogeneous sensor signals in agricultural science. The focus lies on the application of advanced modelling approaches from the domain of machine learning.

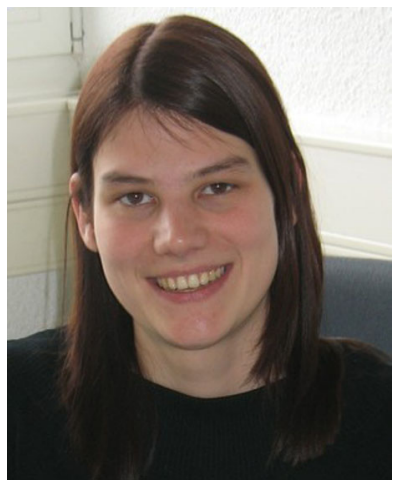

Kathrin Hendriksen studied Agricultural Engineering at Bonn University in Germany until 2008. She worked as product manager for a company producing automatic calf feeders. Currently she is completing her Ph.D. thesis in Bonn. Her research interest is the information technology in the dairy cow management especially the use of new sensor systems and their reliability. 


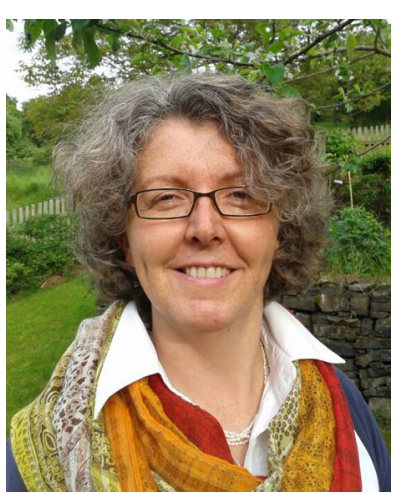

Ute Müller is an Academic Senior Councillor at the Institute of Animal Science at the University of Bonn. She is a lecturer and researcher in the field of strategies for an ecologically keeping of dairy cows in consideration of animal welfare. After she has got the diploma in agricultural science in 1993 she finished her PhD-thesis at Bonn University in 1996. She worked as an agricultural consultant for milk production and herd management and returned to Bonn University in 2000.

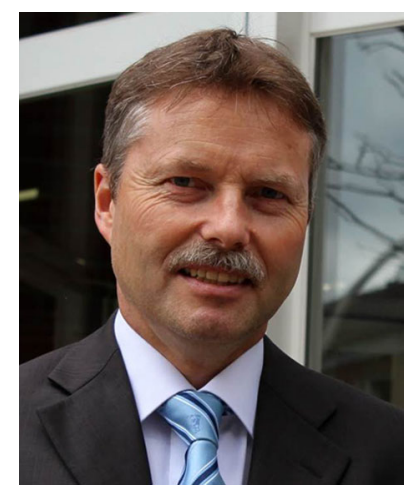

Prof. Wolfgang Büscher is leader of the section „Animal Technology” at the University of Bonn within the Faculty of Agriculture. He is teacher and researcher in the field of animal husbandry with the focus on precision livestock farming. After he has got the diploma in agricultural science he finished his $\mathrm{PhD}$-thesis at Bonn University with the title "Ammonia emissions of forced ventilated animal houses". From 1991 to 1996 he worked as an assistant at Hohenheim University (Stuttgart). His first professorship he has got at Halle University in 1996. In 2001 he returned to Bonn University taking over the current functions. 


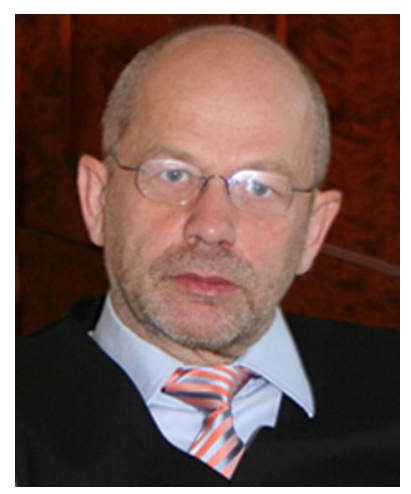

Prof. Lutz Plümer is a full professor at the Institute for Geodesy and Geoinformation, University of Bonn, Germany. He initially studied computer science, received his Master's and Ph.D. degree from the University of Dortmund and his venia legendi from the University of Bonn. His current research focuses on geoinformation, geometric-topological integrity, 3-D models and urban GIS, spatio-temporal data mining and early detection of stress in plants. 